\title{
Research on Algorithm Design and Implementation of Counting Steps Based on Mobile Phone Sensor
}

\author{
Wei Wu \\ School of Cryptography, Information Engineering University, Zhengzhou, 450002, China
}

Keywords: Algorithm design and implementation, Counting steps, Mobile phone sensor

\begin{abstract}
Step counting function can be implemented according to the acceleration data collected by appropriate algorithm. Based on the features analysis of intelligent mobile phone sensor, the paper describes the design principle of the algorithm of counting steps, and gives the implementation methods of data acquisition module, data processing module and data display module of the of the step counting algorithm to provide some references for the relevant researchers.
\end{abstract}

\section{Introduction}

With the improvement of people's living standard and health care, walking is widely used as one of the most common and effective ways of exercise. As a commonly used exercise equipment, the pedometer also has a rapid development. From the beginning of the 2D mechanical pedometer to the development of electronic pedometer based on acceleration sensor as the core, to now more integrated smart bracelet, the pedometer accuracy is greatly improved and the user experience is gradually optimized. The intelligent mobile phone has been equipped with a variety of sensors, but also with the current rapid development of communications, microelectronics, integrated circuits and software technology, the rapid popularization of mobile terminal, mobile phone is only a phone function to the development of integrated rich computing, intelligent sensing and communication capabilities of mobile terminal equipment. At the same time, the accuracy and accuracy of mobile sensors have been improved. Smartphones are no longer simply electronic devices because smartphones are constantly improving their computing and using the perception of a variety of sensors. New algorithms and programs can also be used to turn mobile phones into powerful perceptual environments, sensory devices, and tools that can be used in your life. At the same time, because smartphones are smaller than computers, they have portability and freer mobility. Mobile phones are ubiquitous mobile devices, and millions of people use them all over the world. In the past few years, sensors have become more popular in mobile devices by implanting sensors into mobile phones. This paper develops a set of high accuracy and portable intelligent pedometer system based on previous design experience. At the same time, this paper adopts Android intelligent mobile phone as a pedometer computer system to enhance the user experience.

\section{Concept and Features of Mobile Phone Sensor}

Sensor technology plays an increasingly important role in modern science. The sensors are applied in all kinds of advanced equipment and instruments to collect data for scientific analysis, or to make our equipment more intelligent. Because of the development of sensor technology, it has laid a good foundation for the advancement of science and technology and social development. In recent years, based on the traditional sensor technology, a new sensor manufacturing process has been developed. Finally, a new type of sensor is developed, which greatly improves the microelectronic technology. The sensors for measuring acceleration are divided into the following capacitive, resonant and piezoelectric acceleration sensors. Capacitive acceleration sensor: its basic principle is based on the instantaneous change of capacitance to measure the acceleration change, so it has high sensitivity, sensitivity and little influence by the surrounding environment, the advantages of good linearity; 
disadvantages: because of the existing interference to the capacitor, so the accuracy of the interference is large. Resonant acceleration sensor: the principle is that when the acceleration of the object changes, the resonance changes, which will affect the inherent frequency of the object, through the natural frequency changes, so you can measure the size of acceleration. The characteristic is that the output circuit is simple, and it can also be output directly. The disadvantage is the complicated manufacturing process and the great influence of the outside temperature. Its basic working principle is that the acceleration changes when it results in a power mechanical device. The damping effect is used to measure the acceleration changes. It is characterized by simple process, simple measuring method and good linearity. At present, the common acceleration sensors in the mobile phone can sense the motion of the mobile phone, and continuously acquire three components of the three-dimensional coordinate system. The sensor has the characteristics of small size and low power consumption.

\section{Algorithm Design of Counting Steps Based on Mobile Phone Sensor}

Algorithm Principle. The gait of a human body is a periodic process. The gait cycle is the process of moving from one foot to the ground to the foot again. In this process, the body's center of gravity will fluctuate because of alternating bending of the thighs. In addition, people's walking process is not uniform in micro view, but a variable acceleration movement. This algorithm is divided into 4 parts: data channel selection, auxiliary step algorithm, correction factor estimation and body step algorithm. The data channel selection stage is mainly to select the three axis speed data channel to reduce the amount of data processed by the algorithm. In the auxiliary step algorithm stage, the Calman filter and signal amplitude analysis are carried out by selecting a small acceleration signal. The threshold decision method is used to perform the step calculation. This algorithm has higher precision, but it also requires strong computing power and storage capability. The correction factor estimation stage is mainly based on the analysis results of the algorithm and auxiliary step signal peak, get a correction for the subsequent subject step algorithm. The main stage is the main process step algorithm step. It uses adaptive threshold setting a dynamic correction factor for peak judgment. The algorithm using the difference filter to the sampling frequency is low, but its accuracy depending on the step threshold correction factor is closely related with the auxiliary gauge step accuracy step algorithm step. We can not only consider the gait of the user habits and mobile phone display, but also can adapt to different motion state of the user, which has strong robustness.

Algorithm Design. The accelerometers built in mobile phones are typically three axes. It can measure the forward acceleration, lateral acceleration and vertical acceleration when the mobile phone is moving. During the motion, the amplitude of lateral acceleration is relatively weak, and has no periodic characteristics. Therefore, it is not necessary to consider the partial data when calculating the acceleration amplitude. The lateral acceleration can be obtained by detecting the variance of each set of data. The smallest variance is considered as the lateral data, and all the data is zero. First, the synthetic acceleration data is processed by filtering. The filter has very good results in noise reduction and smoothing. We use the threshold method to determine the peak acceleration, which is easily affected by many factors. The correlation analysis algorithm uses the similarity between the acceleration sequences generated by the pedestrian continuous motion to calculate the number of steps. The method divides the movement state of pedestrians into two types: leisure and walking: leisure, including stillness, standing, sitting, body rotation, basic gesture movements, etc., which do not lead to changes in the position of the personnel. To walk by means of placing a cell phone in a pocket, holding it in the hands, swinging, or using it. The algorithm is divided into two steps in each step: the first is the standard deviation calculation. Second is the autocorrelation calculation. In order to reduce the influence of position of the mobile phone is placed, the algorithm of three axis acceleration signals modulo the overall acceleration velocity with time periodic changes obviously, this cyclical variation is due to pedestrian continuous rhythmic walking produced. The cycle of change may vary in different pedestrian walks, but the walking cycle of the same pedestrian varies 
only within a small range. By using this periodic variation, the autocorrelation of the overall acceleration of the current stride period and the last stride cycle is calculated, and the motion state of the pedestrian is further judged according to the size of the autocorrelation.

\section{Algorithm Implementation of Counting Steps Based on Mobile Phone Sensor}

Module of Data Acquisition. The sensor data acquisition module has been working in the background. From the start of the system, it has been using mobile phone acceleration sensors have been collecting mobile phone data. At the same time, it transfers data to the data display module and data processing module. The module and data display module are modules that need to work all the time, so pay attention to the performance of the module, reduce unnecessary operations and improve performance. The data acquisition module is mainly used to monitor the sensor by registering, and the data can be collected in the sensor, and the data is transmitted back to the data acquisition module and other related display modules through the callback function. Distance measurement module is the module responsible for the specific measurement of the operation process in the system. It needs to interact with the user partly, and has a great deal to do with the data processing module, which directly controls the data processing. When the user presses the button to send the distance and distance measurement test button, data module must have been processing the data collected by sensors. The characteristics of commonly used table is not the behavior of objects and object characteristics of flexion, abstraction results. Compared with data - oriented objects, $\mathrm{W}$ can clearly understand objects and recognize objects by feature description objects. The general case of feature vectors relates to clever observation or indirect observation detection object obtained, characteristic value can make the original data collected by sensors can also be preprocessed data, but the play can better characterize the research object. The data acquisition and distance measurement is two parallel module and distance measurement module by monitoring the callback function measurement, when the measurement starts to the data acquisition module to get the data, the data processing module, until the end of the measurement, the final measurement distance returned to the user, the flow chart as shown below. The user opens the program after going directly into the home page. This sensor has been in the work of the state, has been in the acquisition of three direction acceleration data, and the data collected will be displayed on the mobile phone screen, where the user can see the real-time data waveform of acceleration sensor, when the mobile phone from the static, then press the test button, the system will be in its initial velocity is zero to calculate the moving distance. Move straight along the line to the other end of the measurement. When the button is released, the end of the measurement shows the distance to move. When the user presses the ranging button again, a new range is started.

Module of Data Processing. In the data processing module, the data collected by the algorithm is changed into the physical quantity that we need, and the acceleration from each moment and different direction becomes the physical quantity of displacement that we need. This module is mainly related with the data acquisition module and distance measurement module: distance measurement module and data processing module to support, calculate the displacement of fixed time; and the data processing module, data acquisition module to data support. The data processing module mainly deals with the data collected by the previous acceleration sensors, and calculates the displacement of the time series through a series of acceleration sequences on the time axis. Through the numerical integral, we can determine the initial velocity and initial displacement in the case of a certain period from the initial displacement to the displacement of the end of the displacement. For the calculation of this integral function, we approximate it by matrix integral. The time interval between them shift between two points on a rectangular area, the approximated area is equal to the function itself. In mobile phones, our acceleration sensors have very high sampling frequency, and the average mobile phone has a sampling frequency of thousands. The fusion of the underlying elements combines the initial signal data collected directly by sensors, and then extracts the features of the fused data and makes decisions and judgments. The fusion method has higher requirements for the original data. It can restore the object information to the maximum extent by using the data in the system, and provide the 
high-precision data that other fusion methods cannot provide. The limitations of the fusion are also more obvious, because the sensor data transmitted to the fusion without treatment. Also, a large amount of data processing time is long and the requirements for the system hardware resources is higher, higher requirements for data communication equipment integration at the bottom of the occasion, to solve the sensor data instability and incomplete of fusion also needs to have the high error correction mechanism and processing the original data sources for the same type of sensor. At sufficiently high sampling frequencies, our integrals will have better approximations. In the system, we define the objects in three directions respectively, and deal with the three directions respectively. Finally, we calculate the total displacement by each sub vector, that is, the distance we need to measure.

Module of Data Display. The data display module is an important module in this system. The data display module is responsible for the real-time display in the process of measurement, acceleration, velocity and displacement of each direction of the situation, so that users can also can grasp the change of data in the measurement. In the mobile terminal will always be real-time display of the acceleration sensor data acquisition, the real-time monitor displays three direction acceleration waveform display in the home and changes the history of acceleration, velocity and displacement values simultaneously in three directions of data support. In this module, a new component is abstracted, which is responsible for displaying data using a waveform graph. Through the data display module, the user can observe the data change process in the measurement process at the same time, and can obtain the data of a certain point in the test process. The data display module, which is a common module, will always be used when the system is started, and involves acceleration of the three directions, which we encapsulate as a new class. It provides dynamic addition and reduction of elements, implementation and access to flexible settings, and the size of arrays. It implements all optional list operations and allows all elements within the array. For each instance, there is a capacity that represents the size of the list element. It must be greater than or equal to the size of the list. However, as the element is added, the capacity grows automatically. But the growth strategy is not the addition of elements that will bring about a fixed amount of time spent as jane. Each instance has a capacity. This capacity refers to the size of the array used to store the list elements. It is always at least equal to the size of the list. With the addition of elements to the center, its capacity increases automatically.

\section{Conclusion}

In this paper, the autocorrelation analysis algorithm of the mobile phone acceleration sensor is used to realize the function of step counting. The system works stably and the output data is accurate. The counting result is independent of the human movement condition and the environment. However, in the process of calculation, we only take into account the acceleration information, ignoring the factor of the gyro data. Therefore, the step counting algorithm has the optimized space.

\section{References}

[1] Wang Wenjie, Li Jun. Algorithm Design of Counting Steps Based on Mobile Acceleration Sensor [J]. Industrial Control Computer, 2016, 29(1): 75-79.

[2] Liang Jiuzhen, Zhu Xiangjun, Chen Jing. A high- accuracy and low- sampling- rate step counting algorithm design based on smartphone's accelerometer [J]. Journal of Northwest University (Natural Science Edition), 2015, 45(5): 738-744.

[3] Lou Xizhong, Zhi Li, Fang Jun. Algorithm of MEMS sensor step counting [J]. Journal of China University of Metrology, 2017, 28(1): 81-86.

[4] Xu Bin, Pei Xiaofang, Li Taiyun. Design of Wearable Smart Pedometer [J]. Electronic Science and Technology, 2016, 29(3): 178-182. 